\title{
Thermal relaxation of electron spin motion in a thermal equilibrium ensemble: Relation to paramagnetic nuclear magnetic resonance relaxation
}

\author{
Robert Sharp ${ }^{\text {a) }}$ and Lawrence Lohr \\ Department of Chemistry, The University of Michigan, Ann Arbor, Michigan 48109
}

(Received 28 February 2001; accepted 28 June 2001)

\begin{abstract}
The electron spin relaxation times measured in ESR spectroscopy are physically distinct from the electron spin relaxation times which appear in the theory of NMR Paramagnetic Relaxation Enhancement (NMR-PRE). ESR involves decay of a perturbed spin density matrix toward thermal equilibrium, while in NMR-PRE measurements, the electron spin density matrix remains at thermal equilibrium throughout the NMR experiment. The pertinent spin relaxation involves the thermal decay of the time correlation functions, $\mathrm{G}_{r}(\tau) \equiv\left\langle\mathrm{S}_{r}(0) \cdot \mathrm{S}_{r}(\tau)\right\rangle \quad(r=x, y, z)$, of the spin components, quantities which describe the persistence in microscopic correlation of the spin motion in the thermal equilibrium sample. The decay of the $\mathrm{G}_{r}(\tau)$ is shown to be level-specific; i.e., $\mathrm{G}_{r}(\tau)$ is composed of a sum of contribution associated with individual eigenstates, each of which decays exponentially via a process that is uncoupled to the decay in other eigenstates. This behavior differs markedly from the decay of the nonequilibrium parts of a perturbed density matrix, which involves coupled degree of freedom of the electron spin system. An expression for the level-specific relaxation times has been derived in terms of Redfield matrix elements. This expression is valid for any $S \geqslant 1$ when the static spin Hamiltonian consists of Zeeman and zfs contributions of arbitrary magnitude. Simple closed-form expressions are given for level-specific relaxation times in the cylindrical and orthorhombic zfs limits for $S=1$ and $S=3 / 2$. The theory is used to interpret electron and nuclear spin relaxation for $S=3 / 2$ with specific reference to high-spin $\mathrm{Co}(\mathrm{II})$, for which the zfs splittings are typically large. For this spin system, the presence of orthorhombic terms in the zfs tensor causes profound shortening of the electron spin relaxation times relative to the reference cylindrical zfs case and, in consequence, a comparably large rhombicity-induced depression of the NMR relaxation efficiency. (c) 2001 American Institute of Physics. [DOI: 10.1063/1.1350638]
\end{abstract}

\section{INTRODUCTION}

The $T_{1}$ and $T_{2}$ electron spin relaxation times of ESR spectroscopy describe the decay of an electron spin magnetization vector from a perturbed initial state toward thermal equilibrium. This process is described by Redfield Theory, ${ }^{1,2}$ which derives solutions for the equation of motion of the spin density matrix using second-order time-dependent perturbation theory.

Electron spin relaxation of a somewhat different kind is important in NMR paramagnetic relaxation enhancement (NMR-PRE) phenomena, i.e., enhancements in NMR relaxation rates produced by dissolved paramagnetic solutes. In solutions containing paramagnetic metal ions, quite small concentrations (milli- or microMolar) of metal ion often provide the predominant relaxation pathway for nuclear spins on solvent and ligand species. This phenomenon has been widely used to probe molecular structure, dynamics, and magnetic properties of paramagnetic complexes. The mechanism of the NMR-PRE depends on theoretical parameters usually termed "electron spin relaxation times," although in this experiment, the electron spin density matrix remains at thermal equilibrium (or very nearly so). Thus, electron spin relaxation, as it pertains to NMR-PRE, does not involve decay of a nonequilibrium density matrix, and the relaxation

${ }^{a)}$ Fax: 734-647-4865; electronic mail: rrsharp@umich.edu times of ESR and NMR-PRE experiments are distinct physical quantities, for which we use the symbols, $T_{\mathrm{S}, r}$ and $\tau_{\mathrm{S}, r}$. Though these quantities describe distinct macroscopic phenomena, they result from the same underlying microscopic spin transition.

Electron spin relaxation enters the NMR experiment in the following way. ${ }^{3-5}$ The NMR relaxation mechanism involves stochastic fluctuation of the electron-nuclear hyperfine (HF) interaction, which induces thermal transitions of the nuclear spin. The scalar and dipolar parts of the timedependent HF tensor provide independent relaxation mechanisms. Energy transfer between the electron and nuclear spin systems (S and I) depends on the resonant component of the hyperfine interaction, i.e., it is proportional to the Fourier component of the hyperfine coupling at the nuclear spin transition frequency. Although the coherent motions of $\langle\mathrm{I}\rangle$ and $\langle\mathrm{S}\rangle$ are not resonant, the stochastic motion of the $\mathrm{S}$ spin arising from electron spin relaxation and, in the case of the dipolar HF mechanism, from Brownian reorientation of the interspin I-S vector, introduce a frequency continuum into the hyperfine power spectrum. The HF power peaks, which are centered at the electron spin transition frequencies, are broadened into Lorentzian bands by the thermal motions of S. It is this continuum aspect of the HF power spectrum that provides the resonant Fourier component required for I-S energy transfer and NMR relaxation. Thus, electron spin re- 
laxation times, as they pertain to NMR-PRE, describe linewidths in the HF power spectrum.

The mathematical quantities which describe electron spin motions, both coherent and stochastic, in a thermal equilibrium sample are the time correlation functions (TCFs) of the electron spin components, defined in the Cartesian basis as

$$
\mathrm{G}_{r}(\tau) \equiv \overline{\left\langle\mathrm{S}_{r}(\tau) \cdot \mathrm{S}_{r}(0)\right\rangle} \quad(r=x, y, z) .
$$

An analogous quantity, $\mathrm{G}_{\rho}(t)$, can be defined in the circular basis,

$$
\mathrm{G}_{\rho}(\tau) \equiv\left\langle\mathrm{S}_{\rho}(\tau) \cdot \mathrm{S}_{-\rho}(0)\right\rangle . \quad(\rho=0, \pm 1),
$$

although only the Cartesian functions will be used here. The angle brackets denote a quantum mechanical expectation value, and the superscripting line denotes an average over molecular degrees of freedom, which are usually treated classically. The $\mathrm{G}_{r}(\tau)$ are macroscopic properties of a thermal equilibrium sample, and as such, they are not functions of the absolute time $(t)$, but rather of a time interval $(\tau)$. The $\mathrm{G}_{r}(\tau)$ describe the time dependence of the spin motion in terms of the expectation value of a scalar product, $\left\langle\mathrm{S}_{r}(0)\right.$ $\left.\cdot S_{r}(\tau)\right\rangle$. They are sometimes described as memory functions. When $\tau=0$, the memory represented by the scalar product is perfect, and the TCF has its maximum value,

$$
\mathrm{G}_{r}(0)=\left\langle\mathrm{S}_{r}^{2}\right\rangle .
$$

With increasing $\tau, \mathrm{G}_{r}(\tau)$ in general undergoes coherent oscillation, the frequencies and amplitudes of which are determined by the static spin Hamiltonian. Superimposed on the coherent motion, $\mathrm{G}_{r}(\tau)$ relaxes thermally, and over time, the spin motion loses predictable correlations with its initial state at $\tau=0$ due to spin transitions resulting from the spin's interaction with the thermal lattice. After a long interval, $\mathrm{G}_{r}(\tau) \rightarrow 0$.

The relaxing processes described by the quantities $\tau_{\mathrm{S}, r}$ and $T_{\mathrm{S}, r}$ differ in various ways, the most important of which is that, as is shown below, relaxation of $\mathrm{G}_{r}(\tau)$ is a levelspecific phenomenon; i.e., the decay process within a given spin eigenstate $|\alpha\rangle$ does not couple with the decay in other eigenstates $|\beta\rangle$. Thus, level-specific relaxation times constants, $\tau_{\mathrm{S}, r}^{(\alpha)}$, can be computed for individual eigenstates. This behavior differs from the thermal decay of the nonequilibrium part of the density matrix, in which the relaxing quantities are eigenvectors of the Redfield Matrix, which in general represent coupled degrees of freedom of the spin system. ${ }^{2,6}$ In Density Matrix Theory (DMT), the kinetic equations which describe the decay of the density matrix from a perturbed state toward thermal equilibrium contain cross-terms coupling different density matrix elements. As a result, any given density matrix elements decays via a kinetic process that is in general multiexponential, reflecting a superposition of parallel relaxation modes. In contrast, the TCFs, $\mathrm{G}_{r}(\tau)$, which describe the state of microscopic correlation in the spin motion in a thermal equilibrium sample, decay via a single exponential process within individual spin eigenstates. This result is demonstrated in the following, and the physical reasons for it are discussed.
The macroscopic kinetic equations which describe the decay of $\mathrm{G}_{r}(\tau)$ differ from those describing the decay of a perturbed density matrix. However, the rate constants for both types of relaxation can be expressed in terms of Redfield Matrix elements, which describe the microscopic transition probabilities. In certain limiting cases where the spin Hamiltonian is particularly simple, the numerical values of the decay constants may coincide (examples of this are the longitudinal relaxation times for $S=1$ and $S=3 / 2$ in the cylindrical zfs-limit, see the following) although the physical meaning of the relaxation times differs in all cases.

An interesting point of comparison with our study is provided by recent density matrix calculations ${ }^{6,7}$ of the $T_{\mathrm{S} 1,2}$ relaxation times for $\mathrm{S}=1$ in the zfs-limit. Bertini et al. ${ }^{6}$ have pointed out that in the cylindrical zfs-limit, the Redfield eigenvectors correspond to well-defined tensor components, scalar, dipolar, and quadrupolar, of macroscopic spin order, each associated with a distinct relaxation time. The scalar part of the density matrix is associated with a zero Redfield eigenvalue, reflecting the fact that the trace of the density matrix does not decay. The $\tau_{\mathrm{S}, r}^{(\alpha)}$, in contrast, describe an essentially microscopic relaxation phenomenon involving randomization of the motions of individual spins. Contributions to $\mathrm{G}_{r}(\tau)$ arise from all parts of the density matrix including the scalar component, which is, in fact, the largest contributor to the TCF as well as to the NMR-PRE. The $\tau_{\mathrm{S}, r}^{(\alpha)}$ relaxation process is eigenstate-specific in a thermal equilibrium sample and does not correspond to nonequilibrium tensor components of macroscopic spin order or to spin coherence.

An expression is derived below (Eq. 20) for the quantities $\tau_{\mathrm{S}, r}^{(\alpha)}$ in terms of Redfield Matrix elements. The derivation applied to electron spins $S \geqslant 1$, the coherent motions of which are driven by a static spin Hamiltonian consisting of Zeeman and zfs terms of arbitrary magnitude. From this general result, simple closed-form expressions for $\tau_{\mathrm{S}, r}^{(\alpha)}$ are derived for the cases of $S=1$ in the cylindrical and orthorhombic zfs-limits, and for $S=3 / 2$ in the cylindrical zfs-limit. These formulas are given in the Appendix.

The theory is used to interpret electron and nuclear spin relaxation for $S=3 / 2$ with specific reference to high-spin $\mathrm{Co}(\mathrm{II})$, for which the zfs splittings are typically large. ${ }^{8,9}$ It is shown that for this spin system, the presence of orthorhombic terms in the zfs tensor causes profound shortening of the electron spin relaxation times relative to the reference cylindrical zfs case. In consequence, the zfs-limit NMR-PRE of Co(II) is profoundly depressed by the effects of zfs orthorhombicity. A superficially similar phenomenon has previously been predicted ${ }^{10-13}$ and demonstrated experimentally ${ }^{14-18}$ for integer spin systems $(S=1$ and $S=2)$. However, the rhombicity-induced depression of the NMR-PRE that occurs for integer spins results form an entirely different physical mechanism than that for half-integer spins. We describe the situation for $S=3 / 2$ in detail in terms of power plots of the spin motion. These plots provide a direct and intuitive interpretation of the NMR-PRE mechanism ${ }^{14-18}$ (Ref. 5 reviews this area). 


\section{MOTION OF THE TCF's}

In a thermal equilibrium sample, the time correlation functions, $\mathrm{G}_{r}(\tau)$, in Eq. (1a) can be evaluated as the trace,

$$
\mathrm{G}_{r}(\tau)=\operatorname{Tr}\left\{\rho_{\mathrm{S}} \overline{\mathrm{S}_{r}(\tau) \cdot \mathrm{S}_{r}(0)}\right\},
$$

where $\rho_{\mathrm{S}}$ is the density operator of S. In a thermal equilibrium sample, $\left\langle\rho_{\mathrm{S}}\right\rangle$ is diagonal in the spin eigenbasis, and Eq. (3a) evaluates to

$$
\begin{aligned}
\mathrm{G}_{r}(\tau) & =\sum_{\alpha}\left(\rho^{o}\right)_{\alpha \alpha} \sum_{\alpha^{\prime}} \overline{\left\langle\alpha\left|\mathrm{S}_{r}(\tau)\right| \alpha^{\prime}\right\rangle\left\langle\alpha^{\prime}\left|\mathrm{S}_{r}(0)\right| \alpha\right\rangle} \\
& =\sum_{\alpha} \mathrm{G}_{r}^{(\alpha)}(\tau) .
\end{aligned}
$$

Thus $\mathrm{G}_{r}(\tau)$ is composed of a sum of level-specific contributions. We show next that when the spin Hamiltonian, $\mathrm{H}_{S}$, can be written as a sum of static and stochastic terms,

$$
\mathrm{H}_{\mathrm{S}}=\mathrm{H}_{\mathrm{S}}^{(\mathrm{o})}+\mathrm{H}_{\mathrm{S}}^{\prime}(t) \text {, }
$$

and $\mathrm{G}_{r}^{(\alpha)}(\tau)$ have the form

$$
\begin{aligned}
\mathrm{G}_{r}^{(\alpha)}(\tau)= & \left(\rho^{o}\right)_{\alpha \alpha} \\
& \times \exp \left(-\tau / \tau_{\mathrm{S}, r}^{(\alpha)}\right) \sum_{\beta}\left|\left\langle\alpha\left|\mathrm{S}_{r}\right| \beta\right\rangle\right|^{2} \exp \left(-i \omega_{\alpha \beta} \tau\right) .
\end{aligned}
$$

Thus $\mathrm{G}_{\mathrm{r}}^{(\alpha)}(\tau)$ consists of a sum of contributions, each of which oscillates at a transition frequency, $\omega_{\alpha \beta}$, of the electron spin system (including $\omega=0$ for $\alpha=\beta$ ) and decays thermally as described by the time constants, $\tau_{\mathrm{S}, r}^{(\alpha)}$. The static Hamiltonian, $\mathrm{H}_{\mathrm{S}}^{(o)}$, is the sum of a Zeeman term, $\mathrm{H}_{\mathrm{Z}}$, plus a permanent (vibrationally-averaged) zfs term.

$$
\mathrm{H}_{\mathrm{S}}^{(o)}=\mathrm{H}_{\mathrm{Z}}+H_{\mathrm{zfs}}^{(o)} \text {. }
$$

The presence of a nonzero zfs interaction implies $S \geqslant 1$. In the intermediate regime, $\left(\mathrm{H}_{\mathrm{Z}} \approx \mathrm{H}_{\mathrm{zfs}}^{(o)}\right)$, Eq. (5) must be averaged over molecular orientations. To simplify the notation, we proceed assuming a fixed molecular orientation and defer orientational averaging to the final result.

\section{A. The relaxation mechanism}

Spin relaxation results from the effects of $\mathrm{H}_{\mathrm{S}}^{\prime}(t)$, which is assumed to describe collisionally-induced distortions of the zfs tensor due to Brownian collisions of the solute and solvent. The term $\mathrm{H}_{\mathrm{S}}^{\prime}(t)$ can be expressed in the moleculefixed principal axis system of the permanent zfs tensor as

$$
\begin{aligned}
\hbar^{-1} \mathrm{H}_{\mathrm{S}}^{\prime}(t)= & \sum_{\hat{q}} c_{\hat{q}}(t) \mathrm{S}_{\hat{q}}^{(2)} \\
= & c_{\hat{z}^{2}}(t) \mathrm{S}_{\hat{z}^{2}}+c_{\hat{x}^{2}-\hat{y}^{2}}(t) \mathrm{S}_{\hat{x}^{2}-\hat{y}^{2}} \\
& +c_{\hat{x} \hat{y}}(t) \mathrm{S}_{\hat{x} \hat{y}}+c_{\hat{x} \hat{z}}(t) \mathrm{S}_{\hat{x} \hat{z}}+c_{\hat{y} \hat{z}}(t) \mathrm{S}_{\hat{y} \hat{z}} \\
& +4 \text { th O.T. }(\mathrm{S} \geqslant 2 \text { only }) .
\end{aligned}
$$

Fourth-order zfs terms will be neglected. The spin operators are Cartesian tensors defined as

$$
\begin{aligned}
& \mathrm{S}_{\hat{z}^{2}}=(3 / 2)^{1 / 2}\left(S_{\hat{z}}^{2}-\mathrm{S}(\mathrm{S}+1) / 3\right) \\
& \mathrm{S}_{\hat{x}^{2}-\hat{y}^{2}}=2^{-1 / 2}\left(\mathrm{~S}_{\hat{x}}^{2}-\mathrm{S}_{\hat{y}}^{2}\right),
\end{aligned}
$$

$$
\begin{aligned}
& \mathrm{S}_{\hat{x} \hat{z}}=2^{-1 / 2}\left(\mathrm{~S}_{\hat{x}} \mathrm{~S}_{\hat{z}}+\mathrm{S}_{\hat{z}} \mathrm{~S}_{\hat{x}}\right), \\
& \mathrm{S}_{\hat{y} \hat{z}}=2^{-1 / 2}\left(\mathrm{~S}_{\hat{y}} \mathrm{~S}_{\hat{z}}+\mathrm{S}_{\hat{z}} \mathrm{~S}_{\hat{y}}\right), \\
& \mathrm{S}_{\hat{x} \hat{z}}=2^{-1 / 2}\left(\mathrm{~S}_{\hat{x}} \mathrm{~S}_{\hat{y}}+\mathrm{S}_{\hat{y}} \mathrm{~S}_{\hat{x}}\right) .
\end{aligned}
$$

The coefficients $c_{\hat{q}}(t)$ are stochastic functions of time describing Brownian fluctuations of the zfs Hamiltonian.

The stochastic motions of the zfs tensor have usually been grouped in previous work in three general categories: (1) Brownian reorientation; ${ }^{19-24}$ (2) collisionally-induced distortions of the permanent ${ }^{25} \mathrm{zfs}$ tensor, ${ }^{26-30}$ and (3) stochastic vibrational modulation of the zfs tensor associated with thermal excitation and de-excitation of the normal modes of the metal coordination sphere. ${ }^{31,32}$ The time scales of these motions are generally believed to be well-separated in most cases, ${ }^{33}$ with vibrational relaxation considerably faster (10-100 fs) and reorientation slower (>20 ps) than zfs distortional processes $(1-5 \mathrm{ps})$. When this is so, it is possible to consider the resulting spin relaxation as a sum of "reorientational," "vibrational," and "collisional" mechanisms. In Eq. (7), $\mathrm{H}_{\mathrm{S}}^{\prime}(t)$ is taken to describe the collisional mechanism. Equation (4) implies that molecular reorientation is slow enough that the sample can be viewed as a powder for the purpose of the calculation. In this approximation, the reorientational relaxation contribution involves a separate calculation, which in prior work from our laboratory ${ }^{16,17,34}$ has been carried out by numerical simulation of the Brownian reorientational motion.

The general situation, where the time scales are not wellseparated and where $\mathrm{H}_{\mathrm{zfs}}^{(0)}$ is significant (i.e., outside the Zeeman-limit), is a complex physical problem in which the zero-order spin Hamiltonian, $\mathrm{H}_{\mathrm{zfs}}^{(o)}$, is not static but fluctuates randomly as a result of Brownian reorientation. The fact that the zero-order spin Hamiltonian (i.e., the largest part of $\mathrm{H}_{\mathrm{S}}$ ) is time dependent introduces fundamental complications into the problem in that the spin wave function, matrix elements, and transition frequencies are all stochastic functions of time. A theoretical formulation, based on the Stochastic Liouville Equation (SLE), that is capable of describing the general situation has been developed by Bentis, Kowalewski, Westlund and their co-workers. ${ }^{21,22}$ Within the SLE approach (reviewed in Ref. 4), zfs distortional motions have been modeled as a pseudorotation of the principal axis of a transient zfs tensor, ${ }^{29,35}$ and vibrational excitation/de-excitation has been described using a Smoluchowski diffusion operator. $^{32}$ The SLE formalism provides a powerful platform for describing interferences between the various degrees of freedom, but it is computationally complex. The assumption of separated time scales, which is employed here and in the calculations of Refs. 6 and 7, while less general, appears likely to provide a good description of most practical cases, and it leads to relatively simple closed form expressions for the relaxation rates.

\section{B. Equation of motion of the TCF}

In this section we calculate the equation of motion of the $\mathrm{G}_{r}^{(\alpha)}(\tau)$. In the Heisenberg representation,

$$
\mathrm{S}_{r}(\tau)=e^{i \hbar^{-1} \mathrm{H}_{\mathrm{S}} \tau} \mathrm{S}_{r}(0) e^{-i \hbar^{-1} \mathrm{H}_{\mathrm{S}} \tau} .
$$


The static Hamiltonian, $\mathrm{H}_{\mathrm{S}}^{(o)}$ in Eq. (6), drives coherent spin oscillation. The effects of spin oscillation can be separated form those of thermal decay by transforming to the interaction representation,

$$
\mathrm{S}_{r}(\tau)=e^{i \hbar^{-1} \mathrm{H}_{\mathrm{S}}^{(o)} \tau} \widetilde{\mathrm{S}}_{r}(\tau) e^{-i \hbar^{-1} \mathrm{H}_{\mathrm{S}}^{(o)} \tau} .
$$

The matrix elements of $\mathrm{S}_{r}(\tau)$ and $\widetilde{\mathrm{S}}_{r}(\tau)$, expressed in the eigenbasis of $\mathrm{H}_{\mathrm{S}}^{(o)}$, are related by

$$
\left\langle\alpha\left|\mathrm{S}_{r}(\tau)\right| \alpha^{\prime}\right\rangle=e^{i\left(\alpha-\alpha^{\prime}\right) \tau}\left\langle\alpha\left|\widetilde{\mathrm{S}}_{r}(\tau)\right| \alpha^{\prime}\right\rangle
$$

(the argument of the complex exponential function in Eq. (11) uses the notation, $\alpha \equiv \epsilon_{\alpha} \hbar^{-1}$, with $\epsilon_{\alpha}$ the energy of eigenstate $|\alpha\rangle$ ). From Eq. (3b), the time derivative of $\mathrm{G}_{r}^{(\alpha)}(\tau)$ is

$$
\frac{d \mathrm{G}_{r}^{(\alpha)}}{d \tau}=\left(\rho^{o}\right)_{\alpha \alpha} \sum_{\alpha^{\prime}} \frac{d\left\langle\alpha\left|\mathrm{S}_{r}(\tau)\right| \alpha^{\prime}\right\rangle}{d \tau}\left\langle\alpha^{\prime}\left|\mathrm{S}_{r}\right| \alpha\right\rangle .
$$

From Eq. (11),

$$
\begin{aligned}
\frac{d\left\langle\alpha\left|\mathbf{S}_{r}(\tau)\right| \alpha^{\prime}\right\rangle}{d \tau}= & i\left(\alpha-\alpha^{\prime}\right)\left\langle\alpha\left|\mathrm{S}_{r}(\tau)\right| \alpha^{\prime}\right\rangle \\
& +e^{i\left(\alpha-\alpha^{\prime}\right) \tau} \frac{d\left\langle\alpha\left|\mathrm{S}_{r}(\tau)\right| \alpha^{\prime}\right\rangle}{d \tau} .
\end{aligned}
$$

The motion of the spin component operators is described by the equation of motion,

$$
\frac{d \mathrm{~S}_{r}(t)}{d t}=(i / \hbar)\left[\mathrm{H}_{\mathrm{S}}(t), \mathrm{S}_{r}(t)\right],
$$

which differs from that for the density operator only by a negative sign. Equations of the time dependence of the spin matrix elements can be derived using a procedure analogous to that of Redfield Theory, ${ }^{1,2}$ leading to the result,

$$
\begin{aligned}
& {\left[\frac{d\left\langle\alpha\left|\widetilde{\mathrm{S}}_{r}(\tau)\right| \alpha^{\prime}\right\rangle}{d t}\right]_{0}} \\
& \quad=\sum_{\beta, \beta^{\prime}} \mathrm{R}_{\alpha \alpha^{\prime}, \beta \beta^{\prime}} e^{-i\left(\alpha-\alpha^{\prime}-\beta+\beta^{\prime}\right) \tau}\left\langle\beta\left|\mathrm{S}_{r}(0)\right| \beta^{\prime}\right\rangle,
\end{aligned}
$$

where the subscript on the brackets denotes $\tau=0$. Equation (15) has the same form as the corresponding equation for the density matrix elements except for a change of sign of the argument of the complex exponential factor. Inserting Eqs. (13) and (15) into Eq. (12) and using Eq. (11), gives for the motion of $\mathrm{G}_{r}^{(\alpha)}(\tau)$,

$$
\begin{aligned}
& {\left[\frac{d \mathrm{G}_{r}^{(\alpha)}(\tau)}{d \tau}\right]_{0}} \\
& =\left(\rho^{o}\right)_{\alpha \alpha} \sum_{\alpha^{\prime}} i\left(\alpha-\alpha^{\prime}\right)\left\langle\alpha\left|\mathrm{S}_{r}(\tau)\right| \alpha^{\prime}\right\rangle\left\langle\alpha^{\prime}\left|\mathrm{S}_{r}(0)\right| \alpha\right\rangle \\
& \left.\quad+\sum_{\alpha^{\prime}, \beta, \beta^{\prime}} \mathrm{R}_{\alpha \alpha^{\prime}, \beta \beta^{\prime}}\left\langle\beta\left|S_{r}\right| \beta^{\prime}\right\rangle\left\langle\alpha^{\prime}\left|\mathrm{S}_{r}\right| \alpha\right\rangle\right] .
\end{aligned}
$$

The first term on the r.h.s. describes the coherent oscillations of $\mathrm{G}_{r}^{(\alpha)}(\tau)$. Spin relaxation is described by the second term, from which the quantity, $\mathrm{G}_{r}^{(\alpha)}(\tau)$, which exhibits motion only due to thermal relaxation, is defined,

$$
\begin{aligned}
{\left[\frac{d \widetilde{\mathrm{G}}_{r}^{(\alpha)}(\tau)}{d \tau}\right]_{0}=} & \left(\rho^{o}\right) \alpha \alpha \sum_{\alpha^{\prime}, \beta \beta^{\prime}} R_{\alpha \alpha^{\prime}, \beta \beta^{\prime}}\left\langle\beta\left|\mathrm{S}_{r}\right| \beta^{\prime}\right\rangle \\
& \times\left\langle\alpha^{\prime}\left|\mathrm{S}_{r}\right| \alpha\right\rangle .
\end{aligned}
$$

Level-specific relaxation times are defined from Eq. (5),

$$
\left(\tau_{\mathrm{S}, r}^{(\alpha)}\right)^{-1}=-\left[\tilde{\mathrm{G}}_{r}^{(\alpha)}(0)\right]^{-1}\left[\frac{d \widetilde{\mathrm{G}}_{r}^{(\alpha)}(\tau)}{d \tau}\right]_{0},
$$

which, with Eq. (17) plus the fact that

$$
\tilde{\mathrm{G}}_{r}^{(\alpha)}(0)=\left(\rho^{o}\right)_{\alpha \alpha}\left\langle\alpha\left|\mathrm{S}_{r}^{2}\right| \alpha\right\rangle,
$$

gives

$\left(\tau_{\mathrm{S}, r}^{(\alpha)}\right)^{-1}$

$$
=\left\langle\alpha\left|\mathrm{S}_{r}^{2}\right| \alpha\right\rangle^{-1} \sum_{\alpha^{\prime}, \beta, \beta^{\prime}} \mathrm{R}_{\alpha \alpha^{\prime}, \beta \beta^{\prime}}\left\langle\beta\left|\mathrm{S}_{r}\right| \beta^{\prime}\right\rangle\left\langle\alpha^{\prime}\left|\mathrm{S}_{r}\right| \alpha\right\rangle .
$$

This result is valid for all $\mathrm{S}$ and for arbitrary magnitudes of the Zeeman and permanent zfs energies. The relaxation times, $\tau_{\mathrm{S}, r}^{(\alpha)}$, describe relaxation along the principal axes ( $r$ $=\hat{x}, \hat{y}, \hat{z})$ of the molecule-fixed permanent zfs tensor. As was pointed out above, outside the Zeeman- and zfs-limit, Eq. (20) must be averaged over molecular orientations.

Using the stochastic Hamiltonian, $\mathrm{H}_{\mathrm{S}}^{\prime}(t)$, of Eq. (7), the Redfield Matrix element can be written

$$
\begin{aligned}
\mathrm{R}_{\alpha \alpha^{\prime}, \beta \beta^{\prime}}= & \sum_{q}\left[\langle \alpha | \mathrm { S } _ { q } | \beta \rangle \langle \beta ^ { \prime } | \mathrm { S } _ { q } | \alpha ^ { \prime } \rangle \left(k_{q}\left(\alpha^{\prime}-\beta^{\prime}\right)+k_{q}(\alpha\right.\right. \\
& -\beta))-\delta_{\alpha^{\prime} \beta^{\prime}} \sum_{\gamma}\left\langle\gamma\left|\mathrm{S}_{q}\right| \beta\right\rangle\left\langle\alpha\left|\mathrm{S}_{q}\right| \gamma\right\rangle k_{q}(\gamma-\beta) \\
& \left.-\delta_{\alpha \beta} \sum_{\gamma}\left\langle\gamma\left|\mathrm{S}_{q}\right| \alpha^{\prime}\right\rangle\left\langle\beta^{\prime}\left|\mathrm{S}_{q}\right| \gamma\right\rangle k_{q}\left(\gamma-\beta^{\prime}\right)\right],
\end{aligned}
$$

where

$$
k_{q, q^{\prime}}(\omega)=2^{-1} \int_{-\infty}^{\infty} \overline{c_{q}(0) c_{q^{\prime}}(t)} \cos (\omega t) d t .
$$

The stochastic motions of the various Cartesian modes are assumed to be uncorrelated to the decay exponentially, so that

$$
\overline{c_{q}(0) c_{q^{\prime}}(t)}=\overline{\left|c_{q}(0)\right|^{2}} e^{-t / \tau_{q}} \delta_{q, q^{\prime}},
$$

giving

$$
k_{q}(\omega)=C_{q} \frac{\tau_{q}}{1+\omega^{2} \tau_{q}^{2}}=C_{q} j_{q}(\omega) .
$$

Equations (21)-(24) allow for the possibility that the meansquared amplitudes, $C_{q}$, and motional correlation times, $\tau_{q}$, of the various distortional modes differ. 


\section{LEVEL-SPECIFIC SPIN RELAXATION}

The spin relaxation described by Eq. (20) has the following noteworthy aspects:

(1) The decay is level-specific; i.e., the decay associated with a specific element of the density matrix is monoexponential and uncoupled to the decays associated with other density matrix elements. Equation (20) does not, of course, describe the decay of the density matrix, which is constant in a thermal equilibrium sample, but rather randomization of spin motions as described by decay of the spin TCF's, $\widetilde{\mathrm{G}}_{r}^{(\alpha)}(\tau)$. Both kinds of decay constant, $T_{\mathrm{S}, r}$ and $\tau_{\mathrm{S}, r}$, depend on the Redfield Matrix elements, which describe the microscopic transition probabilities. The macroscopic rate equations for the two types of relaxation differ, reflecting physical differences in the macroscopic decay processes.

(2) The $\tau_{\mathrm{S}, r}$ relaxation times describe a fundamentally microscopic phenomenon (i.e., persistence in the state of correlation in the microscopic spin motion) which, unlike the $T_{\mathrm{S}, r}$ relaxation times of DMT, is unrelated to specific macroscopic tensor components of spin order or spin coherence. The $\tau_{\mathrm{S}, r}$ decay involves all parts of the density matrix, including particularly the scalar component, which is almost always the largest contributor to $\widetilde{\mathrm{G}}_{r}(\tau)$ as well as to the NMR-PRE. In contrast, $T_{\mathrm{S}, r}$ relaxation of the scalar part of the density matrix is described by the zero eigenvalue of the Redfield Matrix, corresponding to the fact that the trace of the density matrix does not decay in ESR experiments.

We amplify the physics underlying the eigenstatesspecific nature of the decay of $\widetilde{\mathrm{G}}_{r}^{(\alpha)}(\tau)$ with an example. For definiteness, we assume a Zeeman Hamiltonian and $S=1$. It is assumed that individual electron spins $(i)$ are uncoupled with each other, so that $\widetilde{\mathrm{G}}_{r}(\tau)$ equals a sum of single particle TCF's,

$$
\widetilde{\mathrm{G}}_{r}(\tau)=\sum_{i} \widetilde{\mathrm{G}}_{r, i}(\tau)=\sum_{i} \sum_{\alpha} \widetilde{G}_{r, i}^{(\alpha)}(\tau)
$$

We consider the contribution to the sum from a specific spin $j$, which at $\tau=0$ is in the $|+1\rangle_{j}$ eigenstate. At some time $\tau>0$, the spin undergoes a thermal transition, e.g., $\left\{|+1\rangle_{j}\right.$ $\left.\rightarrow|-1\rangle_{j}\right\}$. This transition randomizes the phase of the spin motion in the transverse plane and thus clearly destroys existing microscopic correlation in the spin motion that contributes to $\widetilde{\mathrm{G}}_{x, y}(\tau)$. The same transition results in the change, $\left(m_{\mathrm{s}}=+1\right) \rightarrow\left(m_{\mathrm{s}}=-1\right)$, in $\left\langle S_{z, j}\right\rangle$. It might be asked whether the transition does not transfer spin correlation out of $\widetilde{\mathrm{G}}_{z, j}^{(+1)}(\tau)$ into $\widetilde{\mathrm{G}}_{z, j}^{(-1)}(\tau)$. However, for this particular particle, $\left\langle-1\left|\rho^{o}(0)\right|-1\right\rangle_{j}=0$, and $\widetilde{\mathrm{G}}_{z, j}^{(-1)}(0)=0$. After the transition, $\left\langle-1\left|\rho^{o}(\tau)\right|-1\right\rangle_{j}=1$, while $\widetilde{\mathrm{G}}_{z, j}^{(-1)}(\tau)$ remains equal to zero (the latter because the density matrix element corresponding to the $|-1\rangle_{j}$ spin state vanished at $\left.\tau=0\right) . \widetilde{G}_{z, j}^{(+1)}(\tau)$ describes the persistence of correlation in spin motion across the interval $t=0$ to $t=\tau$. The transition does not create a state of motional correlation corresponding to a nonzero value of $\widetilde{\mathrm{G}}_{z, j}^{(-1)}(\tau)$ when $\widetilde{\mathrm{G}}_{z, j}^{(-1)}(0)=0$. This example illustrates the fact that relaxation transitions in a thermal equilibrium sample destroy existing correlations in the state of spin motion but do not transfer the state of correlation from one eigenstate to another.

\section{A. Limiting behavior}

Equation (20) describes the relaxation times, $\tau_{\mathrm{S}, r}^{(\alpha)}$, defined in the molecule-fixed zfs principal axis system, in the presence of Zeeman-limit, the orientationally-averaged values of $\tau_{\mathrm{S}, \hat{x}}, \tau_{\mathrm{S}, \hat{y}}$, and $\tau_{\mathrm{S}, \hat{z}}$ necessarily coincide. (This is true even if the laboratory frame $\tau_{\mathrm{s} 1}$ and $\tau_{\mathrm{s} 2}$ relaxation times differ, since the molecule-fixed coordinate frame is randomly oriented with respect to the Zeeman field and since, in the Zeeman-limit, the M-F axes are physically equivalent after orientational averaging.) In test calculations, we have examined the Zeeman-limit behavior of Eq. (20) and have confirmed this point; namely, that the $\tau_{\mathrm{S}, \hat{x}}, \tau_{\mathrm{S}, \hat{y}}, \tau_{\mathrm{S}, \hat{z}}$ relaxation rates equal each other and reduce to the average of the laboratory-frame relaxation rates of Bloembergen-Morgan Theory. ${ }^{26}$

Simple zfs-limit formulas for the level-specific relaxation times can be derived from Eq. (20) in certain cases. The formulas for $\mathrm{S}=1$ in the cylindrical and orthorhombic zfs limits are given in the Appendix, along with formulas for $\mathrm{S}=3 / 2$ in the cylindrical zfs-limit. As is noted in the Appendix, relaxation times are undefined for certain polarizations in specific eigenstates. In the cylindrical zfs-limit, for example, $\left\langle 0\left|\mathrm{~S}_{\hat{z}}^{2}\right| 0\right\rangle=0$, and hence relaxation along the $r=\hat{z}$ direction is undefined. In the orthorhombic zfs-limit, $\left\langle X\left|\mathrm{~S}_{\hat{x}}^{2}\right| X\right\rangle=\left\langle Y\left|\mathrm{~S}_{\hat{y}}^{2}\right| Y\right\rangle=\left\langle Z\left|\mathrm{~S}_{\hat{z}}^{2}\right| Z\right\rangle=0$; thus $\mathrm{G}_{\hat{x}}^{(x)}(\tau)=\mathrm{G}_{\hat{y}}^{(y)}(\tau)$ $=\mathrm{G}_{\hat{z}}^{(z)}(\tau)=0$, and the relaxation of these quantities is likewise undefined.

In certain limiting cases where the spin Hamiltonian is particularly simple, the numerical values of the decay constants, $\tau_{\mathrm{S}, r}$ and $T_{\mathrm{s}, r}$, may coincide, although the macroscopic decay processes which they describe differ. An example of this is the cylindrical zfs-limit situation for $S=1$, where the longitudinal decay constant $\left(\tau_{\mathrm{S}, \hat{z}}\right)$ coincides with the dipolar decay constant of DMT, ${ }^{6}$ although the physical significance of the relaxation times differ. We have examined this point in test calculations (described below) which compare the results of Eq. (20) with those of DMT (evaluated in previous work $\left.^{6,7}\right)$. These calculations compared longitudinal and transverse relaxation times for $S=1,3 / 2,2$, and $5 / 2$ in the cylindrical and orthorhombic zfs-limits. The results coincided only for longitudinal relaxation times of $S=1$ and $\mathrm{S}=3 / 2$ in the cylindrical zfs-limit.

MacLachlan ${ }^{36}$ has shown that in the fast motion limit where all $\omega_{\alpha \beta} \tau_{q} \ll 1$, the relaxation times along any spatial direction approach the limiting value of

$$
\left(\tau_{\mathrm{S}}\right)_{o}^{-1}=[4 \mathrm{~S}(\mathrm{~S}+1)-3]\left(\Delta_{t}^{2} / 5\right) \tau_{q} .
$$

McLachlan's $\Delta_{t}^{2}$ is the trace of the mean-squared transient zfs tensor and is equivalent to $\Sigma C_{q}$ in our expressions. We have confirmed by calculation that in the fast motion limit, Eq. (20) reduces to McLachlan's result for $S$ in the range, $1 \leqslant S \leqslant 5 / 2$, as do the zfs-limiting formulas given in the Appendix. 


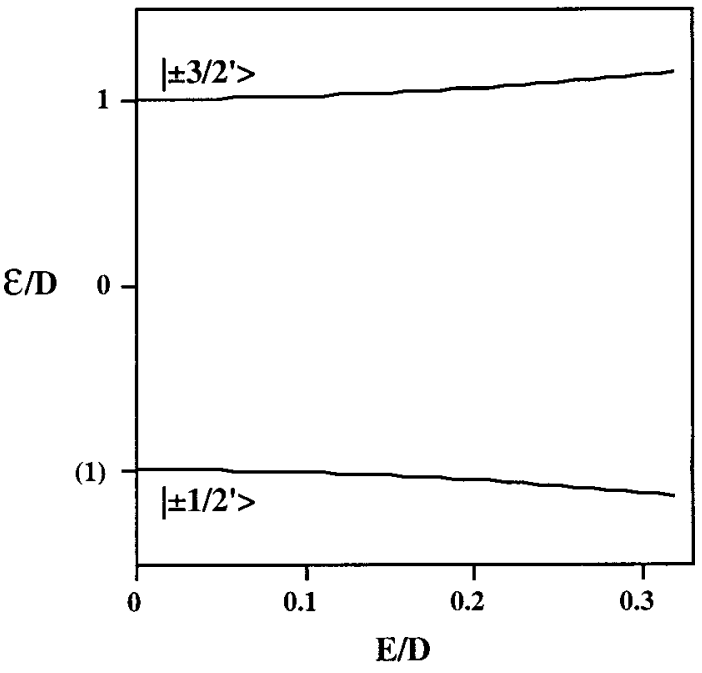

FIG. 1. Energy (in units of D) vs the zfs rhombicity ratio (E/D) for $S=3 / 2$ in the zfs-limit. The wave functions marked correspond to the cylindrical $(\mathrm{E} / \mathrm{D}=0)$ zfs-limit. Nonzero zfs rhombicity mixes eigenfunctions for which $\Delta m_{\mathrm{s}}= \pm 2$.

\section{S=3/2-EFFECTS OF ZFS RHOMBICITY}

This section examines level-specific electron spin relaxation behavior for $S=3 / 2$ and the consequences of this behavior for the NMR-PRE. Spin power plots that are useful for visualizing the NMR relaxation mechanism are described. We specifically consider the case of high-spin $\mathrm{Co}(\mathrm{II})$, an $\mathrm{S}=3 / 2$ ion for which the zfs splittings are characteristically large, ${ }^{8,9} D \approx 10^{2} \mathrm{~cm}^{-1}$, and show that for this ion, zfs orthorhombicity has a very large influence on the electron spin relaxation behavior. As a consequence, the NMR-PRE is likewise strongly affected by zfs rhombicity and may be suppressed by an order of magnitude or more in the orthorhombic complexes relative to the reference cylindrical zfs case. A superficially similar phenomenon involving integer spins has been described theoretically ${ }^{10-13}$ and documented experimentally ${ }^{14-18}$ for both $S=1$ and $S=2$. However, the physical mechanism by which zfs rhombicity exerts its profound effect on the NMR-PRE is very different for integer and half-integer spin systems.

The level diagram (Fig. 1) of $S=3 / 2$ in the zfs-limit consists of two Kramers doublets, the energies of which are, for positive $D$,

$$
\begin{aligned}
& \epsilon^{( \pm 3 / 2)}=\left[D^{2}+3 E^{2}\right]^{1 / 2} \\
& \epsilon^{( \pm 1 / 2)}=-\left[D^{2}+3 E^{2}\right]^{1 / 2} .
\end{aligned}
$$

In the cylindrical zfs-limit, the spin eigenbasis is the circular basis $\left\{\left| \pm 3 / 2^{\prime}\right\rangle\right.$ and $\left.\left| \pm 1 / 2^{\prime}\right\rangle\right\}$ with an axis of spatial quantization parallel to the unique axis of the zfs tensor (the prime denotes a molecule-fixed quantization axis). An orthorhombic component (E) in the zfs tensor does not break the level degeneracies but mixes the eigenstates, $\left|+3 / 2^{\prime}\right\rangle$ with $\left|-1 / 2^{\prime}\right\rangle$ and $\left|+1 / 2^{\prime}\right\rangle$ with $\left|-3 / 2^{\prime}\right\rangle$. The effect of zfs rhombicity on the level energies is modest (Fig. 1), but the effect of rhombicity-induced mixing on the spin wave functions and transition probabilities is large. The effect on the $\left| \pm 3 / 2^{\prime}\right\rangle$ and

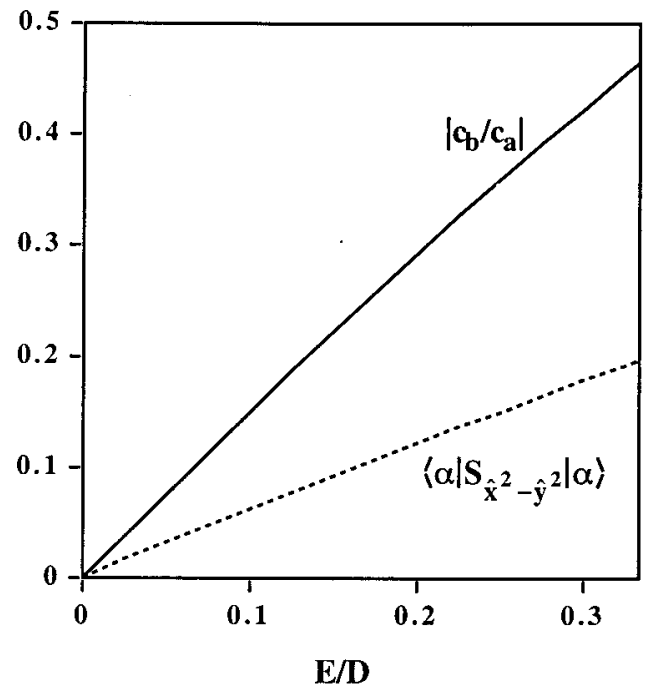

FIG. 2. Mixing of the circular basis functions of $S=3 / 2$ by zfs rhombicity. The ratio of coefficients, $c_{b} / c_{a}$, is defined by Eq. (26a) to the text. E/D is the zfs rhombicity ratio. Also shown (dashed line) is the magnitude of the diagonal matrix elements of $\left\langle\mathrm{S}_{\hat{x}^{2}-\hat{y}^{2}}\right\rangle$.

$\left| \pm 1 / 2^{\prime}\right\rangle$ wave functions is shown in Fig. 2. The wave functions in the orthorhombic zfs-limit can be written

$$
\begin{aligned}
& \left| \pm 3 / 2^{\prime}\right\rangle_{\text {or }}=c_{a}\left| \pm 3 / 2^{\prime}\right\rangle+c_{b}\left|\mp 1 / 2^{\prime}\right\rangle, \\
& \left| \pm 1 / 2^{\prime}\right\rangle_{\text {or }}=c_{a}\left| \pm 1 / 2^{\prime}\right\rangle+c_{b}\left|\mp 3 / 2^{\prime}\right\rangle,
\end{aligned}
$$

Figure 2 shows the variation of the ratio of coefficients, $\left|c_{b} / c_{a}\right|$ versus $\mathrm{E} / \mathrm{D}$ between the cylindrical zfs-limit $(\mathrm{E} / \mathrm{D}=0)$ to the limit of maximum rhombicity $(\mathrm{E} / \mathrm{D}=1 / 3)$. The mixing of the circular basis functions has a profound effect on both the electron spin relaxation times and the NMR-PRE.

The zfs-limit electron spin relaxation times, calculated using Eq. (20), are shown as a function of rhombicity in Fig. 3. The calculations (described in the following) used physi-

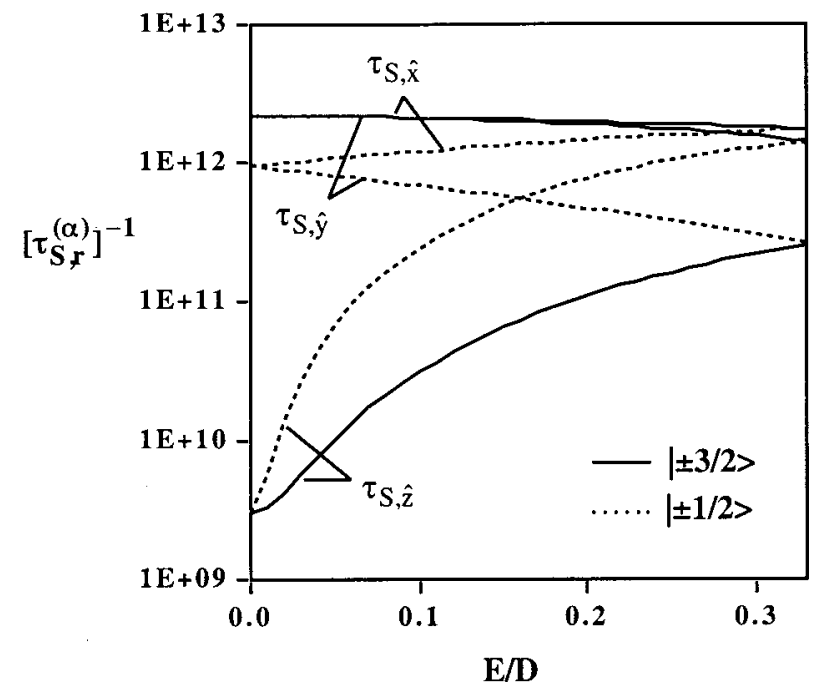

FIG. 3. Level specific relaxation rates, $\left(\tau_{\mathrm{S}, \hat{r}}^{(\alpha)}\right)^{-1}$, versus the zfs rhombicity ratio for $\mathrm{S}=3 / 2$ in the zfs-limit. Calculations are intended as representative for Co(II) with $\mathrm{D}=50 \mathrm{~cm}^{-1}$, all $\tau_{q}=2 \mathrm{ps,} \mathrm{all} C_{q}=5 \mathrm{~cm}^{-2}$. Curves are shown for relaxation along the zfs principal axes, $r=\hat{x}, \hat{y}, \hat{z}$, in the $\left| \pm 1 / 2^{\prime}\right\rangle$ and $\left| \pm 3 / 2^{\prime}\right\rangle$ Kramers doublets. 
cal parameters that are representative of $\mathrm{Co}$ (II) $\mathrm{D}=50 \mathrm{~cm}^{-1}$, $C_{q}=5 \mathrm{~cm}^{-1}, \tau_{q}=2 \mathrm{ps}$. We focus attention on the longest relaxation times, which have the most sensitive influence on the NMR-PRE. (Relaxation times shorter than about 2 ps in the figure violate the Redfield assumption, and for these quantities, calculations based on Eq. (20) are not accurate; for the longer relaxation times, however, the Redfield assumption is amply satisfied.)

In the cylindrical zfs-limit, $\tau_{\mathrm{S}, \hat{z}}^{( \pm 1 / 2)}$ and $\tau_{\mathrm{S}, \hat{z}}^{( \pm 3 / 2)}$ are equal to each other and are much longer than the transverse relaxation times. As is shown in the Appendix, these quantities depend only on $j\left(2 \omega_{\mathrm{D}}\right)$, i.e., on the spectral density functions at the interdoublet transition frequency, quantities which are very small in the case of $\mathrm{Co}(\mathrm{II})$, since $2 \omega_{\mathrm{D}} \tau_{q} \ll 1$. Rhombicity-induced mixing of the wave functions introduces transition probability proportional to $j(0)$, and in consequence of this, $\tau_{\mathrm{S}, \hat{z}}^{(\alpha)}$ shortens dramatically as the rhombicity ratio increases from zero. In the calculations of Fig. $3, \tau_{\mathrm{S}, \hat{z}}^{( \pm 3 / 2)}$ shortens by a factor of about $10^{2}$ and $\tau_{\mathrm{S}, \hat{z}}^{( \pm 1 / 2)}$ by a factor of about $10^{3}$ as E/D rises from zero to its maximum value of $1 / 3$. This shortening of the electron spin relaxation times is reflected in a comparable suppression of the NMR-PRE in the rhombic zfs situation relative to the reference cylindrical case (see the following).

\section{A. Effects of mixing on the transition probabilities}

The fact that the electron spin relaxation along $\hat{z}$ is very inefficient in the cylindrical zfs-limit results from the fact that low frequency [i.e., proportional to $j(0)]$ transition probabilities vanish when $\mathrm{E} / \mathrm{D}=0$. We examine in detail the transitions contributing to $\tau_{\mathrm{S}, \hat{z}}^{(\alpha)}$. The transient Hamiltonian, $\mathrm{H}_{\mathrm{S}}^{\prime}(t)$, contains terms which induce transitions between levels for which $\Delta m_{\mathrm{s}}=0\left(\mathrm{~S}_{\hat{z}^{2}}\right), \Delta m_{\mathrm{s}}= \pm 1\left(\mathrm{~S}_{\hat{x} \hat{z}}, \mathrm{~S}_{\hat{y} \hat{z}}\right)$, and $\Delta m_{\mathrm{s}}= \pm 2\left(\mathrm{~S}_{\hat{x} \hat{y}}, \mathrm{~S}_{\hat{x}^{2}-\hat{y}^{2}}\right)$. Transitions coupling $\left|+3 / 2^{\prime}\right\rangle$ with $\left|-3 / 2^{\prime}\right\rangle$ are disallowed in second order time-dependent perturbation theory. The $\Delta m_{\mathrm{s}}= \pm 2$ transitions coupling the $\left|+3 / 2^{\prime}\right\rangle$ and $\left|\mp 1 / 2^{\prime}\right\rangle$ levels have probabilities proportional to $j\left(2 \omega_{\mathrm{D}}\right)$, which is small when $2 \omega_{\mathrm{D}}$ is large. The $\Delta m_{\mathrm{s}}= \pm 1$ transition coupling $\left| \pm 1 / 2^{\prime}\right\rangle$ with $\left|\mp 1 / 2^{\prime}\right\rangle$ have probabilities proportional to $j(0)$. However, in the zfs-limit (both cylindrical and orthorhombic), the matrix elements $\langle \pm 1 / 2| S_{\hat{x} \hat{z}}$ $|\mp 1 / 2\rangle$, and $\left\langle \pm 1 / 2\left|S_{\hat{y} \hat{z}}\right| \mp 1 / 2\right\rangle$, vanish, and hence likewise the probabilities of the intradoublet transitions within the $m_{\mathrm{S}}= \pm 1 / 2$ doublet manifold. Although $\left\langle\mathrm{S}_{\hat{z}^{2}}\right\rangle$ is diagonal, its matrix elements contribute only to $\tau_{\mathrm{S}, \hat{x}}^{(\alpha)}$ and $\tau_{\mathrm{S}, \hat{y}}^{(\alpha)}$, not to $\tau_{\mathrm{S}, \hat{z}}^{(\alpha)}$ (because $\left[\left(\mathrm{S}_{\hat{z}^{2}}, \mathrm{~S}_{\hat{z}}\right]=0\right)$. Thus the only nonvanishing transition probabilities contributing to $\tau_{\mathrm{S}, \hat{z}}^{(\alpha)}$ in the cylindrical zfslimit are for interdoublet transitions, for which the associated spectral density functions are small. For Co(II), where 2D is characteristically very large, the interdoublet transition probabilities are very small and $\tau_{\mathrm{S}, \hat{z}}^{(\alpha)}$ correspondingly long.

The presence of zfs rhombicity changes this situation qualitatively by mixing levels for which $\Delta m_{\mathrm{s}}= \pm 2$ (i.e., $\left| \pm 3 / 2^{\prime}\right\rangle$ with $\left.\left| \pm 1 / 2^{\prime}\right\rangle\right)$, with the result that the transition probabilities proportional to $j(0)$ no longer vanish. Specifically, the mixing creates diagonal matrix elements in $\left\langle\mathrm{S}_{\hat{x}^{2}-\hat{y}^{2}}\right\rangle$ which provide significant zero frequency transition probability for relaxation along $\hat{z}$. The dashed line of Fig. 2

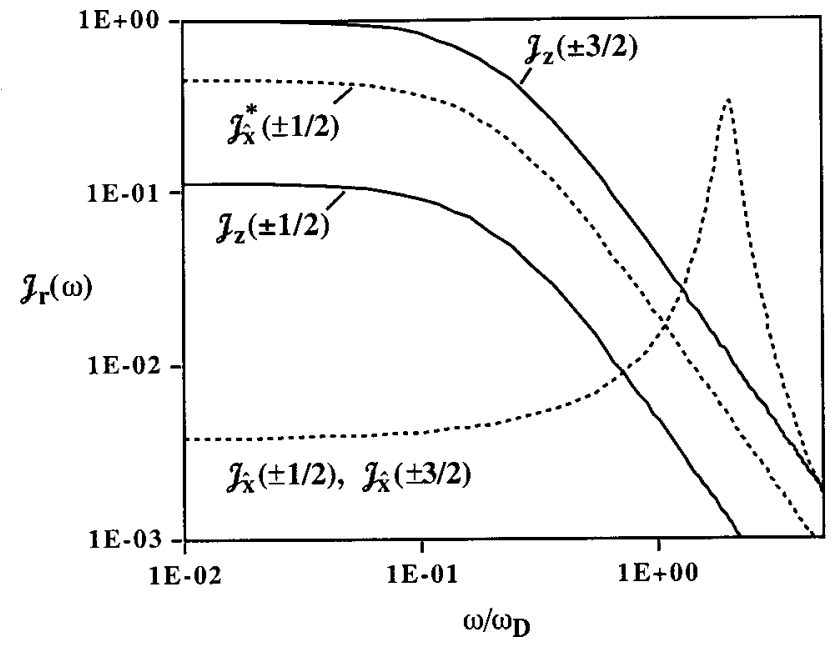

FIG. 4. Power spectra of the spin motion for $S=3 / 2$ in the cylindrical zfs-limit. The calculations assume that all levels have the same electron spin relaxation time, $\tau_{\mathrm{S}, \hat{r}}^{(\alpha)}=20 / \omega_{\mathrm{D}}$. Calculated values are normalized to $\mathscr{J}_{\hat{z}}^{( \pm 3 / 2)}(0)=1$

shows the magnitude of the diagonal matrix elements of $\left\langle\mathrm{S}_{\hat{x}^{2}-\hat{y}^{2}}\right\rangle$ as a function of the zfs rhombicity ratio (all the diagonal elements have equal magnitude). The diagonal ele-

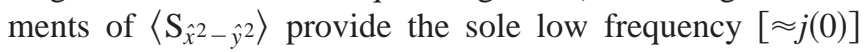
pathway in the zfs-limit. These matrix elements are nonvanishing only when the zfs tensor contains orthorhombic elements.

\section{B. Effects on the NMR-PRE}

To describe the effect on the NMR-PRE, it is useful to consider the motional properties of the $\mathrm{G}_{r}^{(\alpha)}(\tau)$ in the form of power spectra, ${ }^{5,14,15}$ i.e., plots of the cosine transforms

$$
\mathscr{J}_{r}^{(\alpha)}(\omega)=\int_{0}^{\infty} \mathrm{G}_{r}^{(\alpha)}(\tau) \cos (\omega \tau) d \tau
$$

Figure 4 shows the power plots for $S=3 / 2$ in the cylindrical zfs-limit, calculated assuming initially, for the purpose of comparison, that all electron spin relaxation times are equal, $\tau_{\mathrm{S}, \hat{r}}^{(\alpha)}=20 / \omega_{\mathrm{D}}$. In the slow reorientation situation, NMR relaxation efficiency is proportional to the zero frequency power density (or more precisely, to the power density at $\omega$ $=\omega_{I}$ for $T_{1}$ relaxation and at $\omega=0, \omega_{I}$ for $\left.T_{2}\right)$. The power terms labeled $\mathscr{J}_{\hat{z}}^{( \pm 1 / 2)}$ and $\mathscr{D}_{\hat{z}}^{( \pm 3 / 2)}$ arise from diagonal matrix elements of $\left\langle\mathrm{S}_{\hat{z}}\right\rangle$ and are centered at zero frequency. The widths of the power bands are given by the inverse electron spin relaxation times, which are assumed in these calculations, as stated above, to be equal. There are two transverse power bands, labeled $\mathscr{J}_{\hat{x}}^{(\alpha)}$, both of which arise from offdiagonal matrix elements of $\left\langle\mathrm{S}_{\hat{x}}\right\rangle$. One of these, that associated with the matrix elements, $\left\langle \pm 3 / 2^{\prime}\left|S_{\hat{x}}\right| \pm 1 / 2^{\prime}\right\rangle$, connects levels of different Kramers doublets and is centered at the interdoublet transition frequency, $2 \omega_{\mathrm{D}}$. The other (marked with an asterisk) arises from $\left\langle \pm 1 / 2^{\prime}\left|\mathrm{S}_{\hat{x}}\right| \mp 1 / 2^{\prime}\right\rangle$, and is centered at zero frequency (since the levels connected are degenerate). The NMR relaxation efficiency, which is proportional to the power at zero frequency, is determined largely by $\mathscr{J}_{\hat{z}}^{( \pm 3 / 2)}$ and $\mathscr{J}_{\hat{x}}^{*( \pm 1 / 2)}$. The contribution from $\mathscr{J}_{\hat{z}}^{( \pm 1 / 2)}$ is 


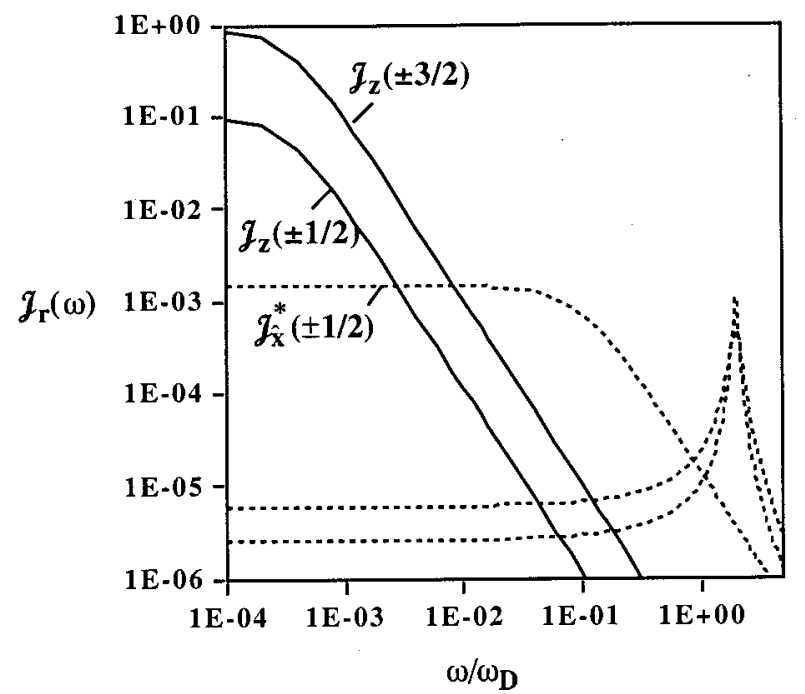

FIG. 5. Power spectra of the spin motion for $S=3 / 2$ in the cylindrical zfs-limit. The calculations, which are intended to be representative of highspin $\mathrm{Co}(\mathrm{II})$, employ level-specific electron spin relaxation times, calculated from Eq. (20) in the text assuming the values of D, $\tau_{q}, C_{q}$ given in the legend of Fig. 3. The calculations are normalized to $\mathscr{J}_{z}^{ \pm 3 / 2)}(0)=1$.

smaller but not insignificant, $1 / 9$ that of $\mathscr{J}_{\hat{z}}^{( \pm 3 / 2)}$. The longitudinal $(\hat{z})$ and transverse $(\hat{x}, \hat{y})$ power bands produce contributions to the NMR relaxation efficiency that have different orientation dependence, an experimental aspect that is described further in the following.

Figure 5 shows a corresponding power plot in which the $\tau_{\mathrm{S}, \hat{r}}^{(\alpha)}$, no longer assumed equal, have been computed using Eq. (20) [this of course provides a much more realistic description of $\mathrm{Co}(\mathrm{II})]$. Because $\tau_{\mathrm{S}, \hat{x}}^{( \pm 1 / 2)} \ll \tau_{\mathrm{S}, \hat{z}}^{( \pm 3 / 2)}$ (see Fig. 3), the transverse contribution of the NMR-PRE is strongly suppressed. In the cylindrical zfs-limit, $\tau_{\mathrm{S}, \hat{z}}^{( \pm 1 / 2)}=\tau_{\mathrm{S}, \hat{z}}^{( \pm 3 / 2)}$, and we thus expect that, since $\mathscr{J}_{\hat{z}}^{(\alpha)}(0) \propto m_{\mathrm{s}}^{2}, 9 / 10$ of th NMR relaxation efficiency arises from $\mathscr{J}_{\hat{z}}^{( \pm 3 / 2)}$ and 1/10 from $\mathscr{J}_{\hat{z}}^{ \pm 1 / 2)}$, with no significant transverse contribution. In this (more realistic) description of the cylindrical zfs-limit, the orientation dependence of the NMR-PRE is entirely longitudinal in character (see the following).

The presence of zfs rhombicity changes this picture in two main ways. First, as described above, $\tau_{\mathrm{S}, \hat{z}}^{( \pm 3 / 2)}$ decreases rapidly as rhombicity increases, and thus the magnitude of the NMR-PRE likewise decreases rapidly with increasing E/D. Second, one of the static transverse contributions, $\mathscr{D}_{\hat{z}}^{*( \pm 3 / 2)}$, that arising from the matrix elements, $\langle \pm 1 / 2| S_{\hat{y}}$ $\mp 1 / 2\rangle$, increases in magnitude with increasing rhombicity as $\mathscr{D}_{z}^{*}( \pm 3 / 2)$ decreases, such that the quantities become equal at $\mathrm{E} / \mathrm{D}=1 / 3$. Thus in the strongly orthorhombic zfs-limit, significant transverse and longitudinal terms contribute to the NMR-PRE. A representative power plot computed for E/D $=0.2$ is shown in Fig. 6. While in the orthorhombic situation the spin matrices are relatively complex, the NMR-PRE results almost entirely from just two terms, one longitudinal $\left(\mathscr{J}_{\hat{z}}^{( \pm 3 / 2)}\right)$, the other transverse $\left(\mathscr{J}_{\hat{y}}^{*( \pm 1 / 2)}\right)$. This situation results form the fact that, as shown in Fig. 3, $\tau_{\mathrm{S}, \hat{y}}^{( \pm 1 / 2)}$ and $\tau_{\mathrm{S}, \hat{z}}^{( \pm 3 / 2)}$

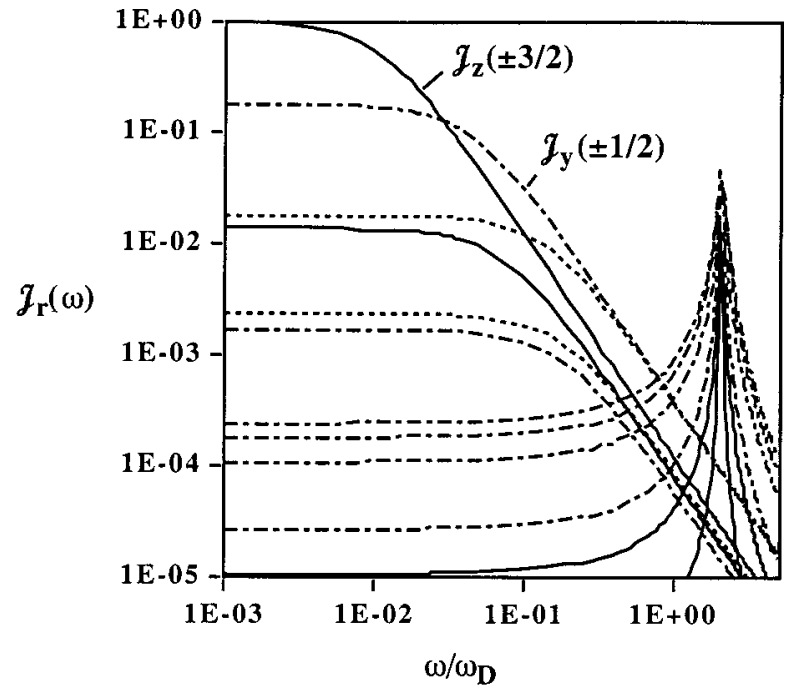

FIG. 6. Power spectra of the spin motion for $S=3 / 2$ in the orthorhombic zfs-limit. The calculations are as described in the legend of Fig. 5 except $\mathrm{E} / \mathrm{D}=0.2$. Solid lines are the contributions to $\mathscr{J}_{\hat{z}}(\omega)$, dashes are contributions to $\mathscr{J}_{\hat{x}}(\omega)$, dash-dot are contributions to $\mathscr{J}_{\hat{y}}(\omega)$.

have comparable magnitude in a strongly orthorhombic zfs field $(\mathrm{E} / \mathrm{D} \approx 1 / 3)$.

In summary, high-spin Co(II) complexes with cylindrical zfs symmetry are predicted to exhibit much higher NMR relaxivity than comparable orthorhombic complexes. However, the influence of electron spin relaxation on the dipolar NMR-PRE is masked by Brownian reorientation when $\tau_{\mathrm{R}}$ $<\tau_{\mathrm{S}, r}$, and thus the large predicted lengthening in $\tau_{\mathrm{S}, r}$ will be reflected much more prominently in the NMR-PRE of slowly reorienting, rather than rapidly reorienting, solutes. The NMR relaxation properties of high-spin Co(II) have been studied in numerous orthorhombic complexes and the inferred electron spin relaxation times are, as expected, generally very short, the order of a picosecond. ${ }^{3,37}$ Experiments involving high-spin Co(II) in sites of cylindrical zfs symmetry have not, to our knowledge, been reported, in part reflecting the fact that $\mathrm{Co}$ (II) porphyrinates tend to be low spin species. ${ }^{38}$ An experimental study of high-spin Co(II) in a cylindrical zfs environment that is reorientationallyimmmobilized [e.g., a Co(II) tetraazamacrocycle complex immobilized in a gel or dissolved in a high viscosity solvent] would obviously be interesting in the present context.

Nilsson and Kowalewski ${ }^{39}$ have used the SLE formalism to calculate the effects of zfs rhombicity on the NMR-PRE for $S=3 / 2$. In their calculations, the introduction of zfs rhombicity produced a mild depression $(\approx 30 \%)$ of the zfs-limit NMR-PRE that is much smaller than the profound ( $>2$ orders of magnitude) rhombicity-induced depression of zfslimit $\tau_{\mathrm{S}, \hat{z}}$ relaxation rate shown in Fig. 3. In part, this difference in result is due to differences in the static zfs parameter, which were much smaller in the calculation of $\mathrm{N}-\mathrm{K}(\mathrm{D} \leqslant 10$ $\left.\mathrm{cm}^{-1}\right)$ than in ours $\left(\mathrm{D}=50 \mathrm{~cm}^{-1}\right)$. However, the major difference probably results from the effects of Brownian reorientation: $\mathrm{N}-\mathrm{K}$ assumed $\tau_{\mathrm{R}}=70 \mathrm{ps}$, while our calculations assume the slow reorientation limit. Reorientation affects the NMR-PRE through modulation of th interspin I-S vector as well as through modulation of the permanent zfs interaction, 


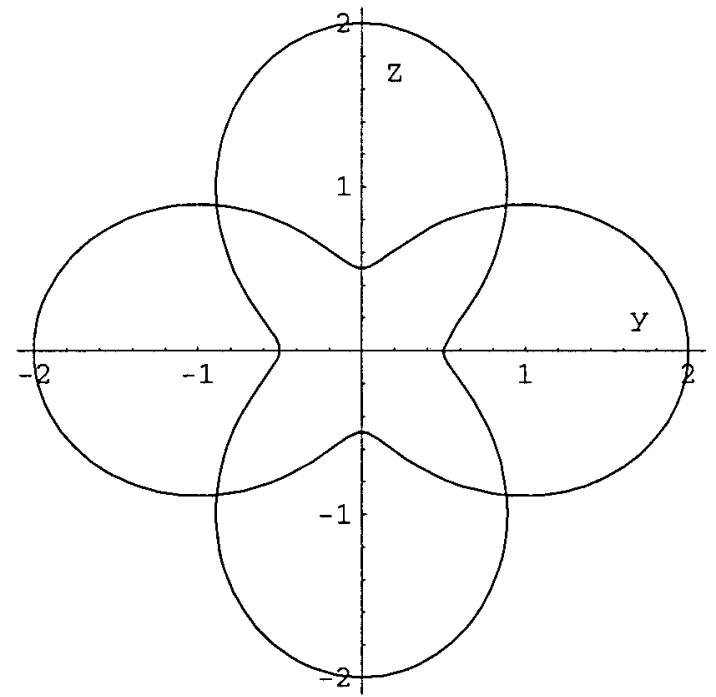

FIG. 7. Polar plots of the angular functions, $\Phi\left(\theta_{\hat{r}}\right)$, for $r=\hat{y}$ and $\hat{z}$.

the latter contributing a mechanism of electron spin relaxation. Both processes contribute to the dipolar correlation time and will tend to mask the large rhombicity-induced lengthening of $\tau_{\mathrm{S}, \hat{z}}$ that is evident in Fig. 3.

\section{Orientational dependence of the NMR-PRE}

Another aspect of the zfs-limit NMR-PRE that is described by the spin power plots concerns the orientational dependence of the NMR $T_{1}$, i.e., the dependence of th NMR $T_{1(2)}$ on the orientation of the I-S interspin vector with respect to the zfs principal axis system. It has been shown ${ }^{14}$ that the angular dependence of the zfs-limit NMR-PRE produced by the power term $\mathscr{J}_{r}^{(\alpha)}$ is the same as that of the mean-squared dipolar field produced by a classical magnetic dipole located at the origin and directed along $\hat{r}$. The mathematical function which describes this dependence is

$$
\Phi\left(\theta_{\hat{r}}\right)=\left[1+\mathrm{P}_{2}\left(\cos \theta_{\hat{r}}\right)\right]
$$

where $\theta_{\hat{r}}$ is the angle between the I-S vector and $\hat{r}$, and $\mathrm{P}_{2}(x)$ is the second order Legendre Polynomial. Polar plots of the functions, $\Phi\left(\theta_{\hat{r}}\right)$, have a characteristic bi-lobed shape shown in Fig. 7, for which the axial/equatorial ratio, $\Phi(0) / \Phi(\pi / 2)$, equals 4 . Each term, $\mathscr{J}_{\hat{r}}^{(\alpha)}$, of the spin power plots contributes NMR relaxation efficiency with a spatial dependence described by the corresponding angular function, $\Phi\left(\theta_{\hat{r}}\right)$; i.e., the transverse power terms, $\mathscr{J}_{\hat{x}}^{(\alpha)}$, produces an NMR-PRE, corrected to constant interspin distance, that is largest for nuclei located on the $\hat{x}$ molecular axis, while the relaxation efficiency produced by $\mathscr{J}_{\hat{z}}^{(\alpha)}$ is largest along $\hat{z}$, etc.

In a previous experimental study, ${ }^{37}$ we reported the orientation dependence of the zfs-limit NMR-PRE for the $\mathrm{S}=3 / 2$ complex, $\mathrm{Co}(\mathrm{II})\left(\mathrm{H}_{2} \mathrm{O}\right)_{2}(\text { acac })_{2}$. The measured (axial/ equatorial) proton NMR $T_{1}$ ratio, corrected to constant interspin distance, was $2.7 \pm 0.4$. Based on this result, it was concluded that the observed NMR-PRE results from competing longitudinal and transverse power terms, the former about five times larger than the latter. The calculations of Figs. 3 and 6 give a more quantitative framework for interpreting this and similar experiments than was available to us previously. As shown in Fig. 6, the observed result occurs at a rhombicity ratio of $\mathrm{E} / \mathrm{D} \approx 0.2$.

\section{Computational procedures}

Calculations of electron spin relaxation times used routines which are incorporated in the computer program, which ParRelax. 2 has been written in this laboratory. ${ }^{5,17}$ This program, which incorporates and supercedes earlier versions, PARELAX, ${ }^{40}$ and RotJmpDyn.f, implements theory of the NMR-PRE at various levels of sophistication. These include (1) Zeeman-limited (SBM) Theory; (2) the slowreorientation theory of the zfs-limit, (3) slow-reorientation theory of the intermediate regime; an (4) Spin-Dynamics simulation techniques ${ }^{16,17,34}$ which describe the effects of Brownian reorientation in the intermediate regime. Each level of theory has both advantages and limitations and should be selected as appropriate for specific problems. The program also contains algorithms which evaluate electron spin relation times using Eq. (20). It is available to the scientific community upon request.

The calculations of Fig. 3 were performed in the following way. At each plotted value of E/D, the zfs-limit Hamiltonian for $S=3 / 2$ was diagonalized. Using the spin eigenfunctions, the matrices $\left\langle\mathrm{S}_{r}\right\rangle$ and $\left\langle\mathrm{S}_{q}\right\rangle$ that are needed for evaluation of Eqs. (20) and (21) were computed in the spin eigenbasis. The spectral density functions $k\left(\omega_{\alpha \beta}\right)$, where computed from the transition frequencies. The relaxation times of Eq. (20) were evaluated using these quantities.

The calculations of Fig. 3 involve the zfs-limit physical situation, where spatial averaging over molecular orientations is not required. In the intermediate regime (where both a Zeeman and a permanent zfs interaction are present), Eq. (20) must be averaged over molecular orientations. The averaging algorithms used in ParRelax.2 performs this average by orienting the Zeeman field with respect to the zfs principal axis system at 92 sampled orientations defined by the polar angles corresponding to the 60 vertices and 32 face centers of the truncated isocahedron (buckeyball). This procedure provides highly effective, unbiased averaging.

\section{CONCLUSIONS}

(1) The electron spin relaxation process which influences NMR paramagnetic relaxation enhancement (NMR-PRE) involve decay of the spin time correlation functions, $\mathrm{G}_{r}(\tau)(r$ $=\hat{x}, \hat{y}, \hat{z})$, defined by Eq. (1), rather than decay of nonequilibrium parts of the spin density matrix. In a thermal equilibrium sample, the decay of $\mathrm{G}_{r}(\tau)$ is level-specific; i.e., $\mathrm{G}_{r}(\tau)$ is composed of a sum of contributions associated with individual eigenstates, each of which decays exponentially via a process that is uncoupled to the decay in other eigenstates.

(2) An expression for the level-specific decay constants has been derived in terms of Redfield matrix elements. This expression [Eq. (20)] is valid for arbitrary $S$ when the spin Hamiltonian consists of Zeeman and zfs contributions of arbitrary magnitude. Spin relaxation is assumed to result 
from collisional modulation of the zfs tensor, when zfs distortion is rapid compared to molecular orientation.

(3) Simple zfs-limit expressions are presented for levelspecific relaxation times of $\mathrm{S}=1$ in the cylindrical and orthorhombic zfs-limits, and of $\mathrm{S}=3 / 2$ in the cylindrical zfs-limit.

(4) The theory is used to interpret electron and nuclear spin relaxation for $S=3 / 2$ with specific reference to highspin $\mathrm{Co}(\mathrm{II})$, for which the zfs splittings are typically large. For this spin system, the presence of orthorhombic terms in the zfs tensor causes profound shortening of the electron spin relaxation times relative to the reference cylindrical zfs case. This phenomenon produces a comparably large rhombicityinduced depression of the NMR relaxation efficiency.

(5) Zfs rhombicity-induced depression of the NMR-PRE has previously been described for integer spin systems $(\mathrm{S}=1$ and $S=2$ ). These latter phenomena, however, originate from a different physical mechanism than that which produces rhombicity-induced depression of the NMR-PRE for halfinteger spins.

\section{APPENDIX}

Level-specific electron sin relaxation times for $S=1$ in the cylindrical and orthorhombic zfs-limits. Relaxation times are defined along the molecule-fixed zfs principal axis directions $(\hat{x}, \hat{y}, \hat{z})$. The quantities $\omega_{\mathrm{D}}, \omega_{\mathrm{E}}$ are the $\mathrm{zfs} \mathrm{D}$, E-parameters in angular frequency units. The Cartesian modes of $\mathrm{H}_{\mathrm{S}}^{\prime}(t)$ are described by a single correlation time, $\tau_{q}$, and mean-square amplitude, $C_{q}$. In the fast motion limit where all $k(\omega) \approx C_{q} \tau_{q}$, all of the following expressions are given by Eq. (25) of the text.

\section{$\mathrm{S}=1$, cylindrical zfs-limit (circular basis):}

$$
\begin{array}{ll}
r=\hat{x}: & \left(\tau_{\mathrm{S}, \hat{x}}^{( \pm 1)}\right)^{-1}=(3 / 2) k_{0}(0)+(5 / 2) k_{1}\left(\omega_{\mathrm{D}}\right)+k_{2}(0) \\
& \tau_{\mathrm{S}, \hat{x}}^{(0)}=\tau_{\mathrm{S}, \hat{x}}^{( \pm 1)} \\
r=\hat{z}: \quad & \left(\tau_{\mathrm{S}, \hat{z}}^{( \pm 1)}\right)^{-1}=4 k_{2}(0)+k_{1}\left(\omega_{\mathrm{D}}\right) \\
& \left(\tau_{\mathrm{S}, \hat{z}}^{(0)}\right)^{-1}=\text { undefined }
\end{array}
$$

$S=1$, orthorhombic zfs-limit (Cartesian basis):

$$
\begin{aligned}
& r=\hat{x}: \quad\left(\tau_{\mathrm{S}, \hat{x}}^{(x)}\right)^{-1}=\text { undefined } \\
& \left(\tau_{\mathrm{S}, \hat{x}}^{(y, z)}\right)^{-1}=2 k(0)+(1 / 2) k\left(2 \omega_{\mathrm{E}}\right)+(1 / 2) k\left(\omega_{\mathrm{D}}-\omega_{\mathrm{E}}\right) \\
& +2 k\left(\omega_{\mathrm{D}}+\omega_{\mathrm{E}}\right) \\
& r=\hat{y}: \quad\left(\tau_{\mathrm{S}, \hat{y}}^{(x, z)}\right)^{-1}=2 k(0)+(1 / 2) k\left(2 \omega_{\mathrm{E}}\right)+2 k\left(\omega_{\mathrm{D}}-\omega_{\mathrm{E}}\right) \\
& +(1 / 2) k\left(\omega_{\mathrm{D}}+\omega_{\mathrm{E}}\right) \\
& \left(\tau_{\mathrm{S}, \hat{y}}^{(y)}\right)^{-1}=\text { undefined } \\
& r=\hat{z}: \quad\left(\tau_{\mathrm{S}, \hat{z}}^{(x, y)}\right)^{-1}=2 k(0)+2 k\left(2 \omega_{\mathrm{E}}\right)+(1 / 2) k\left(\omega_{\mathrm{D}}-\omega_{\mathrm{E}}\right) \\
& +(1 / 2) k\left(\omega_{\mathrm{D}}+\omega_{\mathrm{E}}\right) \\
& \left(\tau_{\mathrm{S}, \hat{z}}^{(z)}\right)^{-1}=\text { undefined }
\end{aligned}
$$

\section{$\mathrm{S}=3 / 2$, cylindrical zfs-limit (circular basis):}

$$
\begin{aligned}
r=\hat{x}: & \left(\tau_{\mathrm{S}, \hat{x}}^{( \pm 3 / 2)}\right)^{-1}=6\left[k(0)+k\left(2 \omega_{\mathrm{D}}\right)\right] \\
& \left(\tau_{\mathrm{S}, \hat{x}}^{( \pm 1 / 2)}\right)^{-1}=7^{-1}\left[18 k(0)+66 k\left(2 \omega_{\mathrm{D}}\right)\right]
\end{aligned}
$$

$$
\begin{array}{ll}
r=\hat{z}: & \left(\tau_{\mathrm{S}, \hat{z}}^{( \pm 3 / 2)}\right)^{-1}=12 k\left(2 \omega_{\mathrm{D}}\right) \\
& \tau_{\mathrm{S}, \hat{z}}^{( \pm 1 / 2)}=\tau_{\mathrm{S}, \hat{z}}^{( \pm 3 / 2)}
\end{array}
$$

${ }^{1}$ A. G. Redfield, Adv. Magn. Reson. 1, 1 (1965).

${ }^{2}$ C. P. Slichter, Principles of Magnetic Resonance, 3 rd ed. (Springer, Berlin, 1990), Ch. 5.

${ }^{3}$ L. Banci, I. Bertini, and C. Luchinat, Nuclear and Electron Relaxation: The Magnetic Nucleus-unpaired Electron Coupling in Solution (VCH, New York, 1991).

${ }^{4}$ P.-O. Westlund, in Dynamics of Solutions and Fluid Mixtures by NMR, edited by J. J. Delpuech (Wiley, New York, 1995), p. 173.

${ }^{5}$ R. Sharp, L. Lohr, and J. Miller, Prog. Nucl. Magn. Reson. Spectrosc. 38, 115 (2001).

${ }^{6}$ I. Bertini, J. Kowalewski, C. Luchinat, T. Nilsson, and G. Parigi, J. Chem. Phys. 111, 5795 (1999).

${ }^{7}$ P.-O. Westlund, J. Chem. Phys. 108, 4945 (1998).

${ }^{8}$ B. N. Figgis, M. Gerloch, J. Lewis, F. E. Mabbs, and G. A. Webb, J. Chem. Soc. A, 2086 (1968).

${ }^{9}$ L. L. Lohr, J. C. Miller, and R. R. Sharp, J. Chem. Phys. 111, 10148 (1999).

${ }^{10}$ H. Fukui, K. Miura, and H. Matsuda, J. Magn. Reson. 88, 311 (1990).

${ }^{11}$ R. R. Sharp, J. Chem. Phys. 98, 6092 (1993).

${ }^{12}$ J.-M. Bovet and R. R. Sharp, J. Chem. Phys. 99, 18 (1993).

${ }^{13}$ T. Nilsson, J. Svoboda, P.-O. Westlund, and J. Kowalewski, J. Chem. Phys. 109, 6364 (1998).

${ }^{14}$ R. Sharp, S. M. Abernathy, and L. L. Lohr, J. Chem. Phys. 107, 7620 (1997).

${ }^{15}$ S. M. Abernathy, J. C. Miller, L. L. Lohr, and R. R. Sharp, J. Chem. Phys. 109, 4035 (1998).

${ }^{16}$ J. Miller, S. Abernathy, and R. Sharp, J. Phys. Chem. A 104, 4839 (2000).

${ }^{17}$ J. C. Miller and R. R. Sharp, J. Phys. Chem. A 104, 4889 (2000).

${ }^{18}$ J. Miller, L. Lohr, and R. Sharp, J. Magn. Reson. 148, 267 (2001).

${ }^{19}$ A. Carrington and G. R. Luckhurst, Mol. Phys. 8, 125 (1964).

${ }^{20}$ D. T. Pegg and D. M. Doddrell, Aust. J. Chem. 29, 1869, 1885 (1976); ibid., 31, 475 (1978).

${ }^{21}$ N. Benetis, J. Kowalewski, L. Nordenskold, H. Wennerstrom, and P.-O. Westlund, Mol. Phys. 48, 329 (1983); ibid., 58, 261 (1984).

${ }^{22}$ N. Benetis and J. Kowalewski, J. Magn. Reson. 65, 13 (1985).

${ }^{23}$ S. Rast, P. H. Fries, and E. Belorizky, J. Chem. Phys. 113, 8724 (2000).

${ }^{24}$ S. Rast, A. Borel, L. Helm, E. Belorizky, P. H. Fries, and A. E. Merbach, J. Am. Chem. Soc. 123, 2637 (2001).

${ }^{25}$ The term "permanent" is used in the same sense as "permanent molecular dipole moment," i.e., averaged over vibrations and over time dependence due to collisional distortion of the zfs tensor.

${ }^{26}$ N. Bloembergen and L. O. Morgan, J. Chem. Phys. 34, 842 (1961).

${ }^{27}$ B. B. Garrett and L. O. Morgan, J. Chem. Phys. 44, 890 (1966).

${ }^{28}$ M. Rubinstein, A. Baram, and Z. Luz, Mol. Phys. 20, 67 (1971).

${ }^{29}$ P.-O. Westlund, N. Benetis, and H. Wennerstrom, Mol. Phys. 61, 177 (1987).

${ }^{30}$ M. Odelius, C. Ribbing, and J. Kowalewski, J. Chem. Phys. 104, 3181 (1996).

${ }^{31}$ S. A. Al'tshuler and K. A. Valiev, Sov. Phys. JETP 35, 661 (1959).

${ }^{32}$ P.-O. Westlund and P. T. Larsson, Acta Chem. Scand. 45, 11 (1991).

${ }^{33}$ Highly detailed molecular dynamics simulations of hexaqua-metal cations, which reorient very rapidly in aqueous solutions, have tended to support this picture; see M. Odelius, C. Ribbing, and J. Kowalewski, J. Chem. Phys. 103, 1800 (1995); J. E. Roberts and J. Schnitker, J. Phys. Chem. 97, 5410 (1993).

${ }^{34}$ S. M. Abernathy and R. R. Sharp, J. Phys. Chem. 101, 3692 (1997).

${ }^{35}$ T. Larsson, P.-O. Westlund, J. Kowalewski, and S. H. Koenig, J. Chem. Phys. 101, 1116 (1994).

${ }^{36}$ A. D. McLachlan, Proc. R. Soc. London A280, 27 (1964).

${ }^{37}$ J. C. Miller, S. M. Abenathy, L. L. Lohr, and R. R. Sharp, J. Phys. Chem. A 104, 9481 (2000).

${ }^{38}$ G. N. La Mar and A. F. Walker, J. Am. Chem. Soc. 95, 1790 (1973).

${ }^{39}$ T. Nilsson and J. Kowalewski, J. Magn. Reson. 146, 345 (2000).

${ }^{40}$ R. R. Sharp, J. Magn. Reson. (1969-1992) 100, 491 (1992). 